\title{
Integration of reinforcement effects over time
}

\author{
JOHN M. HORNER \\ Colorado College, Colorado Springs, Colorado \\ J. E. R. STADDON \\ Duke University, Durham, North Carolina \\ and \\ KAREN K. LOZANO \\ University of Alabama, Birmingham, Alabama
}

\begin{abstract}
Two accounts of how density of reinforcement affects steady-state performance on probabilistic schedules were compared: the real-time linear operator (RTLO) model and a temporal control model (in which response strength is determined by reinforcement probability as a function of postreinforcement time). In Experiment 1, the probability of reinforcement repeatedly cycled between extinction and a random-ratio 10 schedule. Response-rate gain and phase did not change with period of the cycle as predicted by the RTLO model, nor did either model predict the differences in response rate following reinforcement at different points in the cycle. In Experiment 2, the probability of reinforcement was elevated immediately following a reinforcement but fell after a few seconds. Previous reinforcements had no effect upon responding. An extension of the temporal control model, the cumulative impulse model, allowed for the summing of response strength over successive reinforcements and was consistent with the data of both experiments.
\end{abstract}

Many accounts of foraging and behavior on reinforcement schedules (Charnov, 1976; Herrnstein, 1961, 1990; Herrnstein \& Vaughan, 1980) assume that behavior is driven by the animal's estimate of prey density or foodreinforcement rate. How do animals form this estimate from the stream of discrete feeding episodes? Two very different approaches have been taken to this problem. The most popular view in the literature on foraging is that behavior is guided by some form of moving average of local reinforcement rate, referred throughout here as the real-time linear operator (RTLO) model, described by Lea and Dow (1984) but also known as Bush and Mosteller's (1951) linear operator model (see also Bobisud \& Voxman, 1979; Commons, Woodford, Boitano, Ducheny, \& Peck, 1982; Harley, 1981; Herrnstein \& Vaughan, 1980; Kacelnik, Krebs, \& Ens, 1987; Killeen, 1982; Lester, 1984; Myerson \& Miezin, 1980; Ollason, 1980; Pulliam \& Dunford, 1980; Regelmann, 1984).

Another (still popular) approach to this question in the literature on reinforcement schedules appeals to the idea of temporal control. The idea here is that response rate is guided by the probability of reinforcement, assessed as a function of postreinforcement time (Catania, 1970; Catania \& Reynolds, 1968; Gibbon, 1977). We report two experiments that compare the adequacy of these two ac-

This research was supported by grants from the National Science Foundation and the National Institute of Mental Health to Duke University (J.E.R.S. Principal Investigator) and by Colorado College. Correspondence should be addressed to J. M. Horner, Department of Psychology, Colorado College, 14 E. Cache La Poudre, Colorado Springs, CO 80903 (e-mail: jhorner@cc.colorado.edu) counts of steady-state behavior on probabilistic schedules of reinforcement.

\section{The Real-Time Linear Operator Model}

Originally, Bush and Mosteller's (1951) linear operator model was proposed to account for trial-by-trial choice responding. With a simple assumption that "not responding" is an alternative to responding, it can be adapted to temporal responding in real time for a single response. In this RTLO model, response rate is a monotonic function of an animal's estimate of reinforcement density and is not sensitive to anticipated changes in reinforcement probability. The RTLO model assumes that animals maintain an estimate of food density, $P(t)$, which is recalculated during each time step by devaluing the current estimate and adding a fraction of the current input, $I(t)$. The discretetime equation is

$$
P(t+1)=\alpha I(t)+(1-\alpha) P(t), \quad 0 \leq \alpha \leq 1,
$$

where $\alpha$ is a weighting factor that determines the relative influence of the present versus the past and $t+1$ is the current time step. ( $1-\alpha$ may be replaced by a second parameter, $b$, without changing the essential properties of the model.) In foraging or reinforcement-schedule experiments, $I(t)$ represents the effect of a single eating episode at time $t$. Measures of foraging behavior (operant responding, in psychological terminology) are assumed to be directly related to the value of $P$, the system estimate of food density.

The model's sensitivity to changes in food density depends on the value of parameter $\alpha$ : if $\alpha$ is small, the weight given to the current estimate of $P$ (i.e., the integrated ef- 
fect of system history) is large, and $P$ therefore changes only slowly in responses to changes in the density of food, $I$. Conversely, if the weight given to the past is small ( $\alpha$ is large), $P$ changes rapidly with changes in the density of $I$. No matter what the value of $\alpha$, the RTLO model responds in the same way to cyclic changes in food density: if the rate of cyclic change is low, the estimate of food density, $P$, follows variation in the density of food closely, with little phase lag. But if food density varies rapidly, the estimate, $P$, lags behind the actual density and the amplitude of variation in $P$ is reduced.

These two effects of rate of variation in food density are illustrated in Figure 1. Both panels of the figure show the effect on $P$ of a procedure in which food deliveries occur at random, with constant probability, $p=K>0$, for fixed periods of time, alternating with fixed periods when $p=0$ (extinction). The high-low cycle ( $p=K$ vs. $p=0$ ) is shown by the dashed bars; the rising curve shows cumulative responses generated by the model, assuming that during each time step response probability is equal to the model's estimate, $P$, of reinforcement probability. Individual food deliveries are shown by the dots across the middle of the graph.

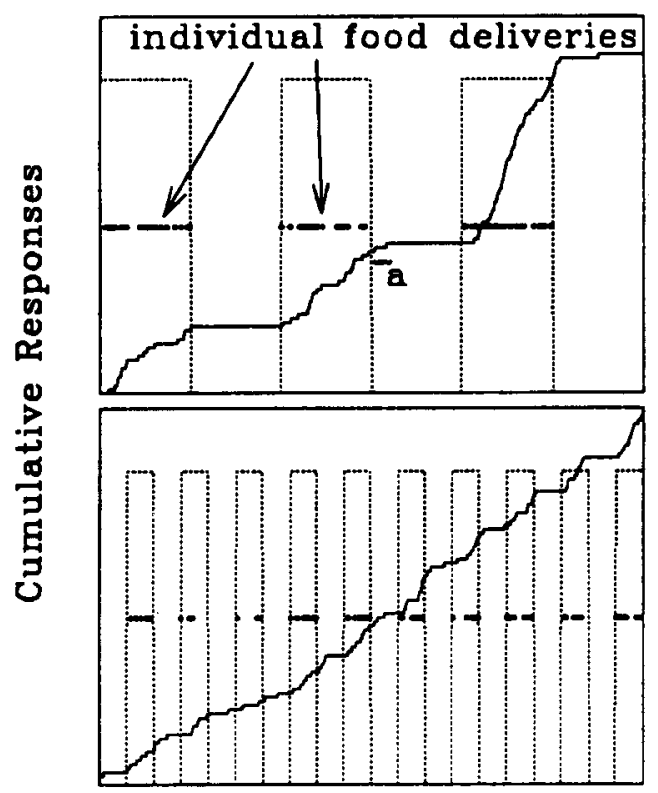

Time

Figure 1. Top panel: how the RTLO model integrates over time. The vertical bars show periods when food probability is either 0 or 0.5 in each time step (period: $M=100$ ). The rising line shows cumulative responses generated by a version of the RTLO model in which response probability in each time step is equal to the model's estimate of food probability, $P(t)$. Points "a" show overrun of responding into extinction periods. Bottom panel: same as above, but for a shorter period input cycle. Note that because these simulations are intended to illustrate the averaging properties of the model, food deliveries are not dependent on responding, as they would be in a real foraging situation. (Parameter $\alpha$ in Equation $1=$ $0.2 ; I(t)=1$ when food is delivered, $I(t)=0$ otherwise.)
Look first at the top panel, which shows a long-period input cycle. Responding closely tracks the cycle: proportionally there is little tendency for responding from the high-probability-of-food part of the cycle to spill over into the extinction part (a period of overshoot is shown by the " $a$ " in the figure) and responding begins early in the high part of each cycle. Although this is harder to see in the figure, when many cycles are averaged, the period of maximum responding almost coincides with the middle of the "high" part of the cycle (i.e., there is only a small phase lag) and responding occurs mostly during the high part (i.e., response amplitude is high). If we denote average response rate during the highest part of the output cycle by $x_{\max }$ and response rate during the lowest part by $x_{\min }$, then amplitude in this context can be defined as the dimensionless quantity $A_{M}=x_{\max } /\left(x_{\max }+x_{\min }\right)$, the proportional change in response rate through the cycle. The subscript $M$ indicates the cycle duration (period). A quantity called gain can be defined as $G(M)=A_{M} / I_{M}$, where $I_{M}$ is the proportional change in food probability between the high and low parts of the cycle, that is, $I_{M}=p_{\max } /$ $\left(p_{\max }+p_{\min }\right)$, which equals unity in the example because $p_{\min }$ is zero. Gain is the ratio of output amplitude to input amplitude.

The bottom panel in Figure 1 shows the effect of a short-period input cycle on the RTLO model. It is clear that the overshoot is necessarily (proportionally) greater than in the top panel, hence the output amplitude, $A_{M}$, is lower and the gain less. Moreover, phase lag, defined as the time difference between response peak and input peak (i.e., the middle of the "high" part of the cycle) and expressed as a fraction of the input cycle, will also be greater when $M$ is less. These two quantities are shown as a function of $M$, the input period, for this version of the RTLO model in Figure 2. As one can see, when the input period increases (frequency decreases), response-rate amplitude (i.e., variation in response rate) increases and phase lag decreases. (While the RTLO model would predict that response-rate amplitude should asymptote for very long periods, it would never predict an actual decrement in amplitude.) Figure 1 also shows that absolute lag (i.e., the simple time difference between input and output peaks) is approximately constant for the RTLO model. A third characteristic, which is also apparent in Figure 1, is that responding should always show a transient increase after each food delivery. These three qualitative properties--decreasing phase lag and increasing gain as period increases and a transient increase in responding after food - do not depend on the value of $\alpha$ and are signatures of the RTLO model and can be easily tested.

\section{Temporal Control}

One of the first discoveries made possible by Skinner's invention of the free-operant method was that rats, pigeons, and many other animals are highly sensitive to the time between food deliveries (Ferster \& Skinner, 1957). For example, if food occurs at fixed intervals, then, after a suitable training period, operant responding begins after 

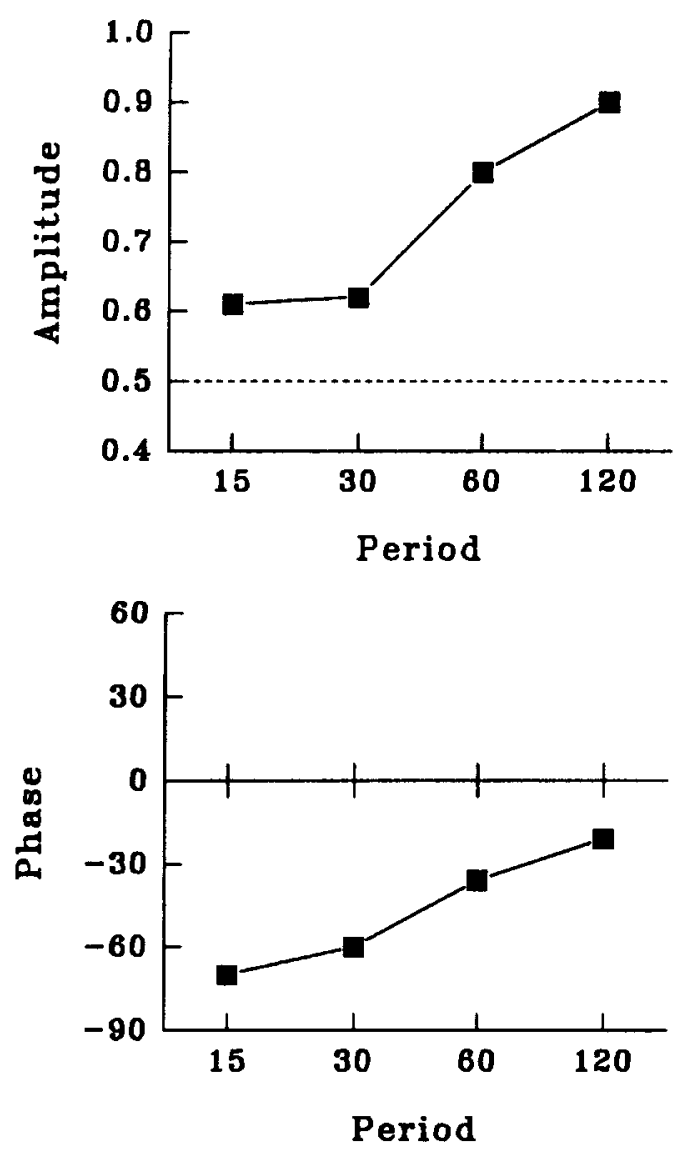

Figure 2. Gain, $G(M)=A_{M} / I_{M}$ (top panel), and phase lag, $\boldsymbol{\theta}(M)$ (bottom panel), as a function of input period, $M$, for the RTLO model. Gain and phase in this figure were computed using the method used later for data (see Figures 4 and 5) rather than the simpler method described in the text. ( $\alpha=.1$ in Equation 1 for these simulations.)

a pause proportional to the interfood interval and increases progressively until the next food delivery (Schneider, 1969). Other early experiments showed that this behavior is triggered by each food delivery, which acts as a sort of time marker that resets the animal's internal clock (Kello, 1972; Mellon, Leak, Fairhurst, \& Gibbon, 1995; Staddon \& Innis, 1966). Catania and Reynolds (1968), and many others subsequently, showed that even if food occurs at irregular intervals, this postfood pattern continues to follow the pattern of reinforcer probability. For example, if there is a small probability of food at time $t_{1}$ after food but food is certain at time $t_{2}$ (where $t_{2} \gg t_{1}$ ), then response rate will rise to a peak at about postfood time $t_{1}$ and then decline before rising to a second peak at $t_{2}$. Only if reinforcer probability is constant (i.e., independent of postfood time), as on random-interval schedules, is there no control of response rate by postfood time.

What postfood pattern of food probability would be associated with cyclical changes in reinforcement probability? Its essential features are obvious. Given equallength $p=.1$ and $p=0$ periods in each cycle, food deliveries (necessarily in the $p=.1$ part of the cycle) must be followed by a period of extinction $M / 2 \mathrm{sec}$ in length, beginning at a postfood time between 0 and $M / 2 \mathrm{sec}$, and depending on where the particular food delivery falls in the $p=.1$ part of the cycle. This period of extinction would then be followed by a period between $M / 2$ and $M \sec$ since reinforcement, where $p=.1$ once again begins, and so on. The average of all these possible profiles is the average postfood profile of food probability.

The postfood profile of food probability is a convolution of response rate and the properties of the cyclical schedule, but it is not strictly determined by response rate, except in the most trivial instance (i.e., not responding) as any response-dependent schedule would be. The periodic nature of the postfood profile of food probability can be accentuated or flattened by changes in the responserate profile, but its periodic nature cannot be fundamentally altered.

Because we wish to account for steady-state behavior on these schedules, we anticipate that these two profiles will have reached some equilibrium state, and hence the postfood pattern of food probability can be measured directly from the actual distribution of interfood delivery times experienced by the animal. However, to obtain a profile that matches the animal's estimate of food probability, we must average this distribution in a way that takes into account limitations on the animal's ability to estimate time intervals. For example, suppose the animal can just detect changes in food probability at postfood time of 10 and $15 \mathrm{sec}$. Because of the Weber-law property of time discrimination (Catania, 1970; Gibbon, 1977; Staddon, 1965), he will therefore be unable to detect changes between (say) 30 and $35 \mathrm{sec}$. Thus, if the temporal grain of our average at $10 \mathrm{sec}$ is $5 \mathrm{sec}$, then at $30 \mathrm{sec}$ it must be $15 \mathrm{sec}$, and so on, proportionately. Therefore, the appropriate estimate of postfood food probability should be computed from the actual distribution of interfood interval, averaged across bins that increase geometrically with postfood time, for example, $1-2,2-4,4-8 \mathrm{sec}$, and so on.

While the details of the postfood profile of food probability for cyclical schedules cannot be estimated until the experiment is completed, the essential feature of a drop in reinforcement probability to $M / 2 \mathrm{sec}$ followed by a recovery at $M \sec$ should produce a reduction in response rates following a reinforcement followed by a subsequent increase in responding. Additionally, this response-rate profile should be sensitive to the length of the cycle period. If, as observed in most periodic schedules, animals anticipate increases in reinforcement, we would expect the animals to synchronize with the cyclical schedule regardless of period length. We might also expect that response-rate amplitude would increase with increase in period length up to a point, as also predicted by RTLO model, but that for long periods, amplitude might deteriorate with temporal discrimination. Lastly, there should be no effect of reinforcement density, or more simply put, of any reinforcements prior to the last one, on response rates.

In Experiment 1, we exposed pigeons to a cyclic probabilistic schedule of the type illustrated in Figure 1. These 
two models, temporal control and the RTLO model, make very different predictions about steady-state behavior under these conditions. We found results inconsistent with the RTLO model and partly consistent with temporal control. In Experiment 2, the probability of reinforcement was elevated immediately following a reinforcement, but fell after a few seconds. This schedule had the effect of making the length of reinforcement bouts and the time between bouts unpredictable. The results of this experiment were inconsistent with the RTLO model but consistent with a modified version of temporal control.

\section{EXPERIMENT 1 Effect of a Cyclic Probabilistic Schedule}

In Experiment 1, hungry pigeons were trained to peck a key for food reinforcement delivered probabilistically. The payoff probability varied cyclically, as in Figure 1. We varied the period of the cycle, $M$, and looked at measures of gain and lag in the steady-state pattern of keypecking by the pigeons. We also looked at the postfood pattern of responding and compared it with the pattern of reinforcement probability as a function of postreinforcement time on this schedule.

\section{Method}

Subjects. Four White Carneaux pigeons (Columba livia), 2 males (No. 04 and No. 08) and 2 females (No. 11 and No. 16), served as subjects. Throughout the experiment the animals were maintained at $85 \%$ of their ad-lib body weights. All animals were housed in individual cages on a 24-h light cycle and given free access to grit and water. All had served previously in operant experiments.

Apparatus. A standard Skinner box, made of aluminum and Plexiglas and measuring $30.5 \mathrm{~cm}$ in length, $24.4 \mathrm{~cm}$ in width, and $27.3 \mathrm{~cm}$ in height, was used. Three response keys were located on an aluminum side wall, but only the center key was used in this experiment. This center key was $2 \mathrm{~cm}$ in diameter and was located in the middle of the wall $19 \mathrm{~cm}$ above the floor. This key was transilluminated by a $28-\mathrm{V}$ white-light bulb during pecking opportunities throughout the session. Each keypeck turned the keylight off for $0.08 \mathrm{sec}$. A food magazine with a $5 \times 5 \mathrm{~cm}$ opening was located $8 \mathrm{~cm}$ above the floor in the middle of the aluminum control panel, directly beneath the center key. During food delivery, the keylight was extinguished and the magazine was illuminated for $2.5 \mathrm{sec}$. The lights from the key and food magazine provided the only illumination during the session. The other three walls and the ceiling of the chamber were made of Plexiglas.

The operant box was enclosed in a larger sound-attenuated chamber. A white-noise source and the noise of the extractor fan masked extraneous sounds. The experiment was controlled by a singleboard computer (Horner, 1991) located in an adjacent room. The computer kept track of each response and reinforcement with accompanying time to within $0.02-\mathrm{sec}$ resolution. Data were transferred to a larger computer for analysis.

Procedure. Throughout the experiment, pecks to the center key were reinforced probabilistically with 2.5 -sec access to mixed grain. The payoff probability for each peck was calculated by comparing the schedule parameter, $p$, with the output of a pseudo-randomnumber generator, $p^{\prime}\left(0-1\right.$ range, rectangular distribution). If $p^{\prime}<p$, the keypeck was reinforced (this procedure is also termed a randomratio $1 / p$ schedule). Reinforcement probability, $p$, varied from condition to condition. All sessions ended when either the pigeon had received 60 reinforcements or $60 \mathrm{~min}$ had elapsed, whichever came first. Sessions typically lasted no more than $30 \mathrm{~min}$, so the time cri- terion was rarely invoked. Sessions were run once a day, usually for 6 days a week.

There were two types of session: (1) baseline, during which pecks were reinforced according to a constant random-ratio 20 ( $\mathrm{RR} 20 ; p=$ .05 ) schedule, (2) and cyclical, in which a $p=.1$ (RR 10) schedule alternated with extinction $(p=0)$ in the way illustrated in Figure 1; cyclical sessions always began with $p=.1$ and the $p=.1$ and $p=0$ periods were of equal duration.

The cycle period, $M$, varied between cyclical conditions. We studied periods of $15,30,60$, and $120 \mathrm{sec}$. To minimize carryover effects, cyclical conditions were separated by baseline conditions; the sequence of cyclical conditions was also counterbalanced. Sequence of conditions for each bird showing the period, $M$ (in seconds), and the number of sessions in each condition are given in Table 1 . All cyclical conditions $(15,30,60$, and 120$)$ had baseline conditions both preceding and following. The number of cyclical and baseline sessions for all conditions is given in the bottom two rows of the table.

\section{Results}

Peck-rate differences between the $p=.1$ and $p=0$ (high and low) periods of the cycle generally increased as period increased, but not in the monotonic way implied by the RTLO model. Figure 3 shows average response rate, in 2 -sec bins, for the 4 pigeons across the cycle under the four cyclical conditions. Cycle amplitude (the difference between the high and low peaks of the response-rate cycle) is small under the 15 -sec condition, larger under the 30- and 60-sec condition, but then generally smaller again under the 120 -sec condition.

The relation between amplitude and period is shown directly in Figure 4. Rather than pick off the maximum and minimum rates from the average curves in Figure 3, which would give too much weight to single data points, we estimated amplitude and phase lag in a way that used all the information in each cycle. We computed the proportion of responses in the high and low parts of the output cycle at zero phase lag: $A(0)=x_{0} /\left(x_{0}+x_{1}\right)$. Thus, for the 30-sec condition, $x_{0}$ is the sum of responses made in Seconds $1-15$, and $x_{1}$ is the sum of responses made in Seconds 16-30 in the average data shown in Figure 3. Then we shifted the averaging window by $1 \mathrm{sec}: A(1)=x_{0}$ ' $\left(x_{0}+x_{1}\right)$, where $x_{0}$ is the sum of responses made in Seconds $2-16$ and $x_{1}$ is the sum of responses made in Seconds 17-30 and 1, and so on. Output amplitude is then defined as the highest value of $A(N)$, and the phase lag, $\Theta$, expressed as a proportion of the period, is $N / M$. Figure 4 shows that, for all 4 pigeons, amplitude increased with period until $M=120$, when amplitude remained constant or decreased for all animals.

Table 1

Sequence of Conditions for Each Bird in Experiment 1

\begin{tabular}{|c|c|c|c|c|c|c|c|c|c|}
\hline \multirow[b]{2}{*}{ Pigeon } & \multicolumn{9}{|c|}{ Sequence of Conditions } \\
\hline & $\overline{B L}$ & I & $\mathrm{BL}$ & 2 & $\mathrm{BL}$ & 3 & BL & 4 & $\mathrm{BL}$ \\
\hline 04 & & 15 & & 120 & & 30 & & 60 & \\
\hline 08 & & 30 & & 15 & & 60 & & 120 & \\
\hline 11 & & 60 & & 30 & & 120 & & 15 & \\
\hline 16 & & 120 & & 60 & & 15 & & 30 & \\
\hline Cyclical & & 14 & & 14 & & 14 & & 14 & \\
\hline Baseline & 3 & & 2 & & 4 & & 4 & & 2 \\
\hline
\end{tabular}

Note-BL, baseline. 


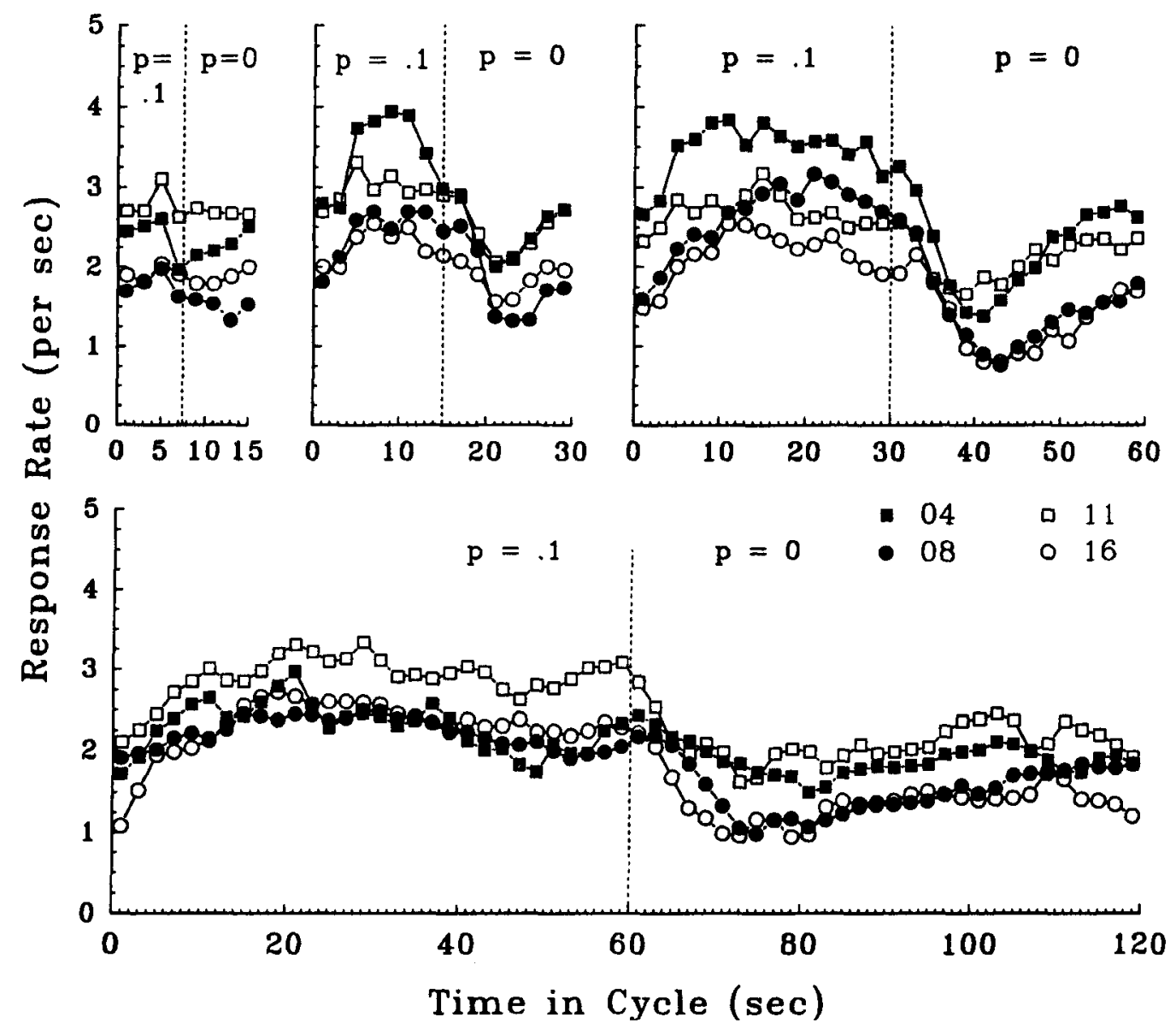

Figure 3. Average response rate across a single cycle for the 4 pigeons and the four $M$ (period) values. The vertical dashed line marks the transition between $p=.1$ (RR 10) and $p=0$ (extinction). (Rates are averages over the last five sessions in each condition.)

Figure 5 shows the effect of period on phase. Phase lag is close to zero for all periods, as is consistent with a temporal control account but not predicted by RTLO.

A final prediction of the RTLO model is that peck rate should be highest immediately after food delivery. Figure 6 shows the average peck rate in 1-sec bins from the onset of food delivery for all 4 pigeons under the baseline (BL) condition and the four $M$ values. Only the BL condition shows an average profile characteristic of the RTLO model: an initial increase followed by a slow decline. Under all four experimental conditions, although there is usually a brief increase in responding right after food and a later decline, the profile almost always shows a second rise later.

Almost every prediction of the RTLO model is violated by our data. Output amplitude does not consistently increase in a monotonic fashion with period; phase lag does not decrease as period increases (there was almost no phase lag at any period), and peck rate does not increase and then decrease after each food delivery. Yet the pigeons are clearly behaving differently during the high and low parts of the input cycle and learning something about the period of the cycle. How do they do it? The temporal- control account provides part of the answer. The cyclic schedule used in this experiment, like any schedule that is not completely random in time, is associated with a postfood pattern of food probability that is not constant. Perhaps our pigeons were adapting to this pattern by generating a postfood profile of pecking more or less matching the postfood profile of changing food probability. Perhaps behavior that appears odd in terms of the RTLO model-no phase lag, a nonmonotonic amplitude versus period function, and so on-is just a by-product of this consistent postfood pattern of keypecking.

Figure 7 shows the postfood probability distributions experienced by the 4 birds during each of the four conditions and baseline in Experiment 1, averaged into geometrically increasing bins as described above. The way that this distribution changes as a function of period $(M$ value) closely resembles the corresponding changes in postfood-response profiles shown in Figure 6. At the shortest $M$ value, both curves show a sharp decline and rapid rise. At the longest $M$ value $(M=120)$, however, both curves show a more or less monotonic decline. Although there are some individual differences, the pattern of change in the response-rate data from the shortest to 


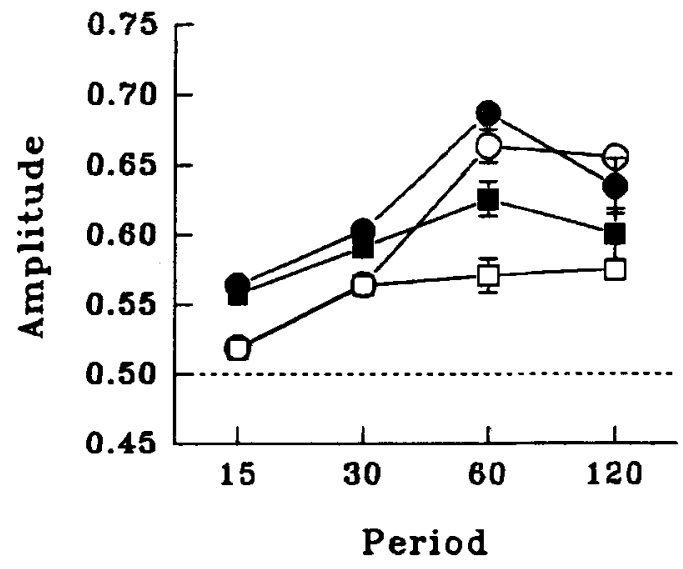

Figure 4. Average response amplitude, $A_{M}$, as a function of period, $M$, for the data in Figure 3. Closed squares represent performance of Pigeon 04; closed circles, Pigeon 08; open squares, Pigeon 11; open circles, Pigeon 16.

the longest $M$ value is well matched by the change in postfood food probability, when postfood food probability is averaged in a way that takes constraints on temporal discrimination into account.

A simulation in which this same fixed pattern of postfood pecking is reliably played out after each food delivery generates average peck-rate data that matches the average data in Figure 3. Figure 8 shows the results of this simulation for 2 animals (Nos. 04 and 08) for the $M=30$ condition. Compare this simulation with the actual data in Figure 3. For both data and simulation, response rates are higher in the $p=.1$ part of the cycle and show the general profile (seen in Figure 6) in the $p=0$ segment of the cycle. What is not well approximated by this simulation for either animal is the anticipatory drop in response rate just before the end of the $p=.1$ part of the cycle. The reason for that will become apparent in a moment.

Figure 9 shows a second feature of the birds' behavior. The figure shows peck-rate versus postfood-time profiles under all cyclic conditions for all 4 animals. This plot is the same kind as that in Figure 6, except that in Figure 9 the first and subsequent food deliveries in each $p=.1$ part of the cycle are plotted separately. The dotted lines show the profiles for the first food delivery in each $p=.1$ part of the cycle. The solid lines show the profiles for the subsequent food deliveries in each $p=.1$ part of the cycle. The pattern after the first food delivery in a cycle is sometimes different from the pattern following subsequent food deliveries. The solid lines almost always show a dip-peck rate decreases for a while after food (showing sensitivity to the upcoming extinction period); the dotted lines in most cases show a brief increase or a slower decrease (showing sensitivity to the upcoming period of reinforcement availability) - see, for example, the $M=30$ condition for Bird 04, the $M=60$ condition for Bird 08, and the $M=120$ condition for Bird 11 . In no case is responding following subsequent food deliveries consistently elevated above that of first food deliveries. The profiles in Figure 9 show some differences among the in- dividual pigeons, but these two features - a systematic change in the average profile as period increases and a systematic difference between the profiles following the first and subsequent food deliveries of each cycle-are common to all.

\section{Discussion}

Temporal control with food as time marker-the idea that each food delivery initiates a fixed peck rate versus postfood-time pattern, and that this pattern is based upon the experienced postfood food probability profileaccounts for the main features of our data. But the systematic differences between the dotted and solid lines in Figure 9 show that it fails to account for reliable secondorder effects of previous reinforcements. How should this difference between the postfood patterns at different points in the cycle be explained?

One possibility is to invoke a new process: discrimination. The idea here is that the pigeons can tell the difference between food deliveries at the beginning of the $p=.1$ part of the cycle (necessarily preceded by at least $M / 2 \mathrm{sec}$ of extinction) and food deliveries later in the $p=.1$ period (necessarily following closely on other food deliveries). Perhaps the pigeons learn that a single food delivery after a period without food signals the onset of the high part of the cycle and thus begin to respond rapidly following any isolated food delivery. The discrimination hypothesis is plausible, but it is also vague and unparsimonious. It requires a new mechanism of discrimination between interfood intervals, but its details are unspecified. We need to rule out simpler possibilities before exploring this hypothesis.

An alternative is a modification of the temporal control idea that retains the basic properties of temporal control while accommodating the differential postfood effects of food deliveries that occur at different points in the cycle. We will consider a modification that allows the effects of one food delivery to extend beyond the next food delivery: the cumulative impulse (CI) model.

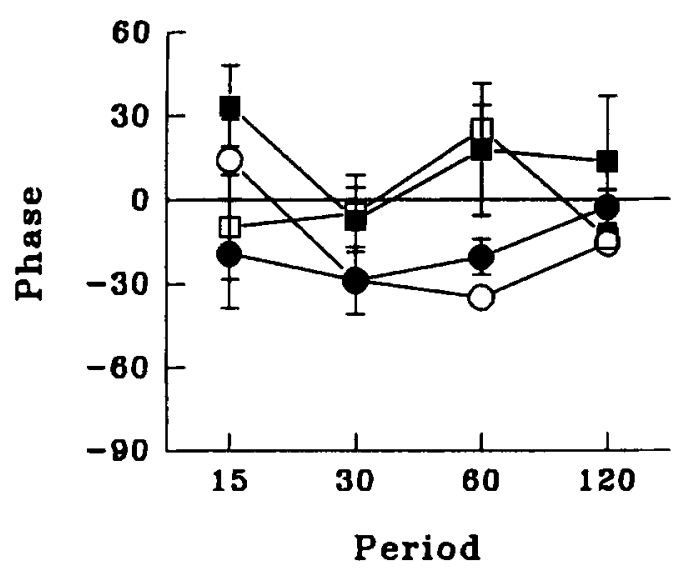

Figure 5. Phase lag, $\theta$ (computed as described in the text) as a function of period, $M$, for the data in Figure 3. Closed squares, Pigeon 04; closed circles, Pigeon 08; open squares, Pigeon 11 ; open circles, Pigeon 16. 


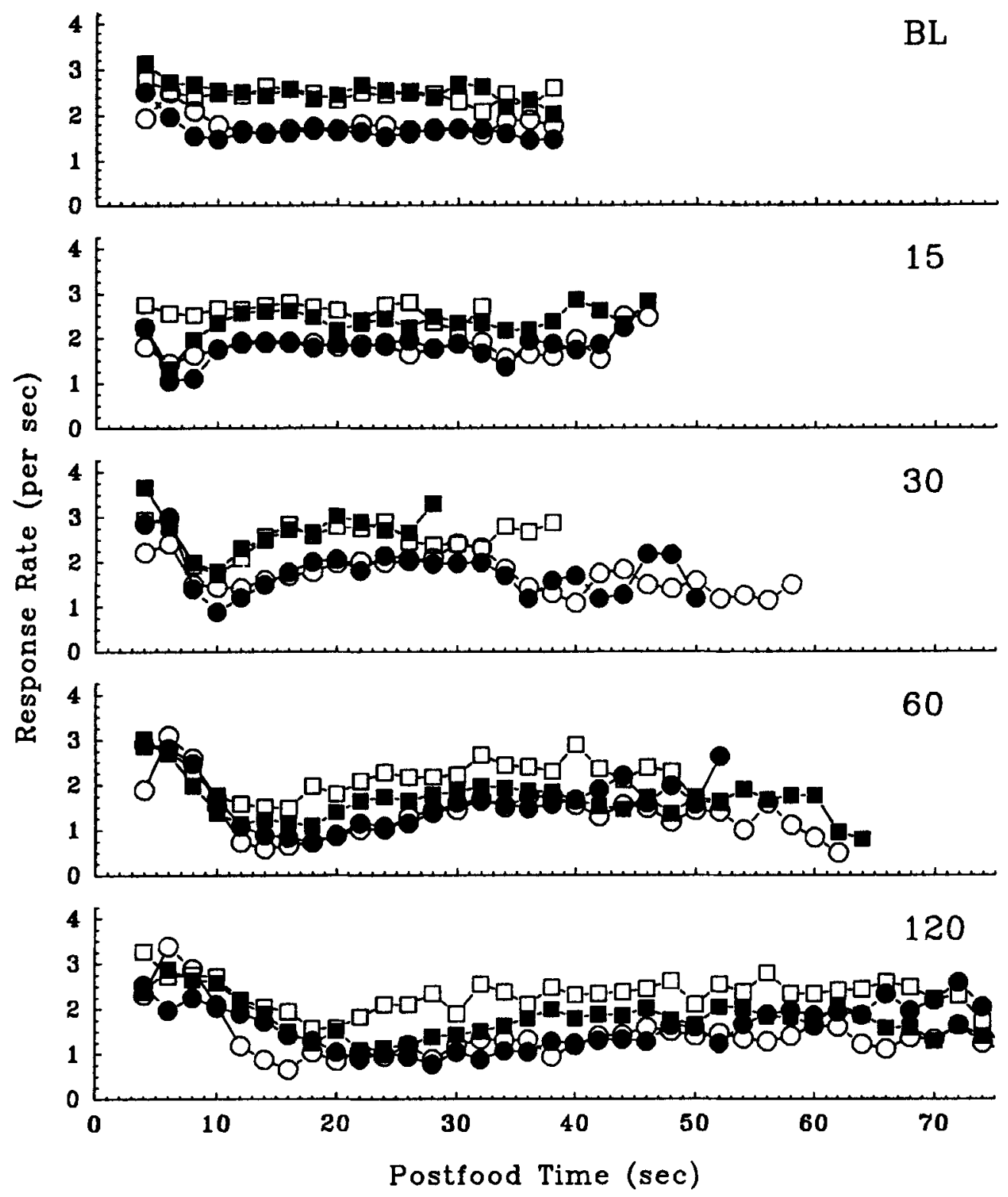

Figure 6. Response rates as a function of postfood time for all animals in all conditions. Each data point represents response rate per second, per opportunity to respond during that postfood time. Postfood time is the time from the onset of food delivery. The first 4 sec of data are omitted to allow for food consumption and the opportunity to return to responding-approximately 3.5 sec. Closed squares, Pigeon 04; closed circles, Pigeon 08; open squares, Pigeon 11; open circles, Pigeon 16. (BL $=$ baseline.)

In the $\mathrm{CI}$ model, the simple temporal control hypothesis can be formalized as follows. Let the most recent food delivery occur at time $t_{0}$; then, at any later time $t$ the response rate, $x(t)$, will be given by a fixed function

$$
x(t)=R\left(t-t_{0}\right), \quad[R(<0)=0] .
$$

$R\left(t-t_{0}\right)$ is just the postfood response profile, examples of which are shown in Figure 6. This model assumes that food deliveries that precede the most recent food delivery have no effect on current behavior. If we relax this assumption and allow previous reinforcements to have a cumulative effect on response strength, we arrive at the CI model, which can be formalized as

$$
\begin{aligned}
x(t)= & a_{0} R\left(t-t_{0}\right)+a_{1} R\left(t-t_{1}\right) \\
& +\ldots+a_{n} R\left(t-t_{n}\right), \quad 0 \leq a i \leq 1,
\end{aligned}
$$

where $R$ is the same fixed postfood response profile as in Equation 2 and the multipliers $a_{i}$ indicate the weighted effects of earlier food deliveries occurring at times $t_{1}, t_{2}$, and so on.

This model thus allows previous reinforcements to affect response rates, but makes the direction and extent of 


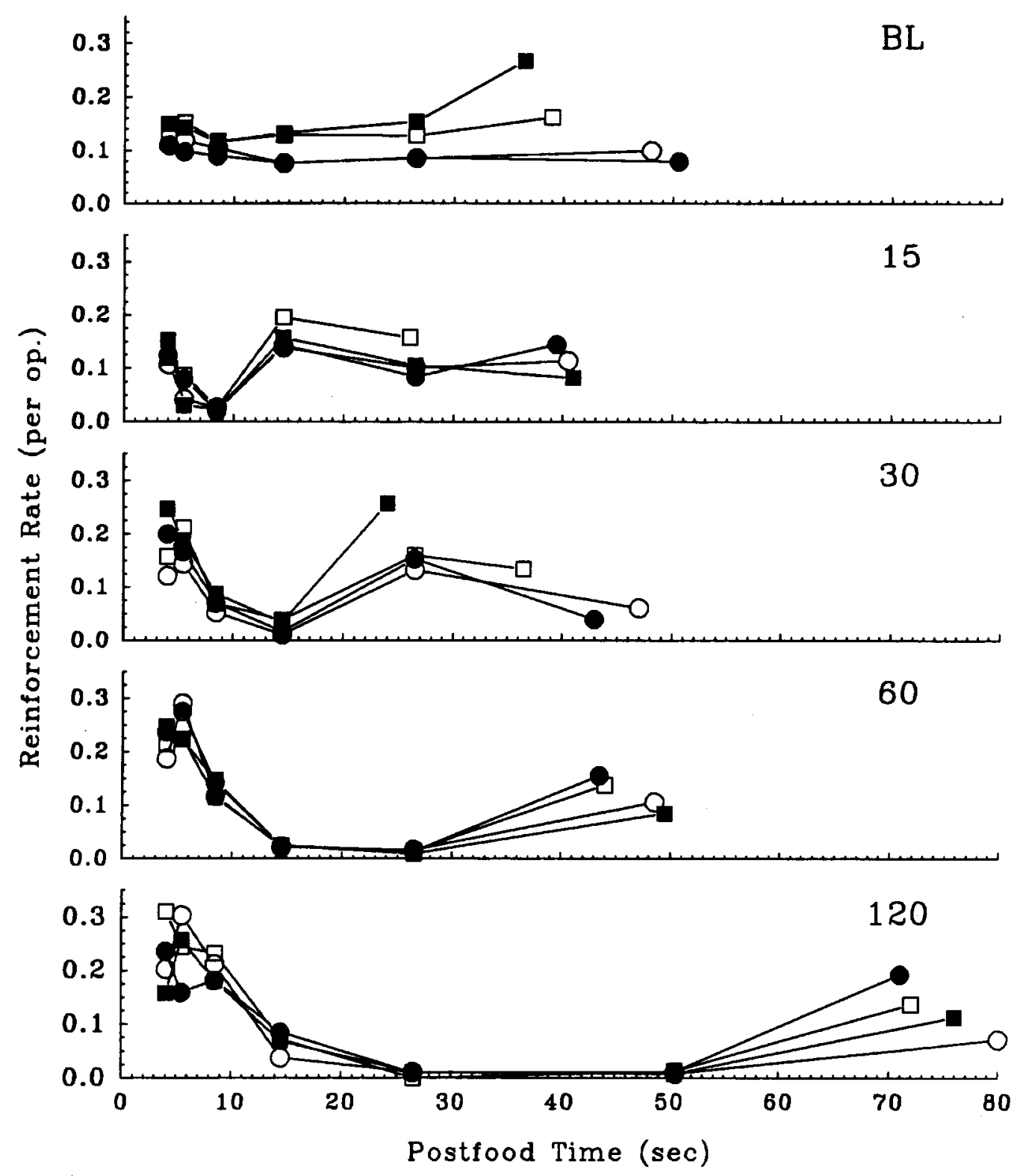

Figure 7. Postfood food-probability distributions (also termed "reinforcement-per-opportunity" distributions in the operant literature) experienced by the 4 birds during the baseline (BL) and each of the four conditions, averaged into geometrically increasing time bins: 3-4, 4-6, 6-10,10-18, 18-34, 34-66, and 66-130 sec. Data points are plotted in the middle of each bin. As in Figure 6, the first 4 sec are not shown. (The last bin in each series is variable in length because of variation in the average number of bins in which the data were available. Thus, for some animals where the data were not complete enough for a geometric-sized final bin, the final bin was shorter.) Closed squares, Pigeon 04; closed circles, Pigeon 08; open squares, Pigeon 11; open circles, Pigeon 16. Compare these postreinforcement probability profiles with the postreinforcement response profiles for each animal in Figure 6.

the effect dependent upon the form of the postfood profile of food probability. Thus, depending upon the overall form of the profile, previous reinforcements could have a facilitatory, inhibitory, or no effect at all. Additionally, the specific effect of any previous reinforcement at any point in time, $t$, would depend upon its timing relative to the last reinforcement $\left(t_{0}-t_{n}\right)$.

The values of $a_{i}$ in this simplified model are used to express the relative weighting of previous food deliver- ies, and thus denote constants in the current model. In exploring the prediction of the CI model $a_{i}$ will be held constant across all food deliveries $\left(a_{0}=a_{1}=a_{2}=C\right)$. In more complex models, $a_{i}$ could vary with $i$ or as a function of time since the reinforcement, $t-t_{i}$.

Allowing reinforcements to have long-range cumulative effects on subsequent responding should produce a counterintuitive effect of reinforcement density in these schedules. Because these schedules have a built-in pe- 


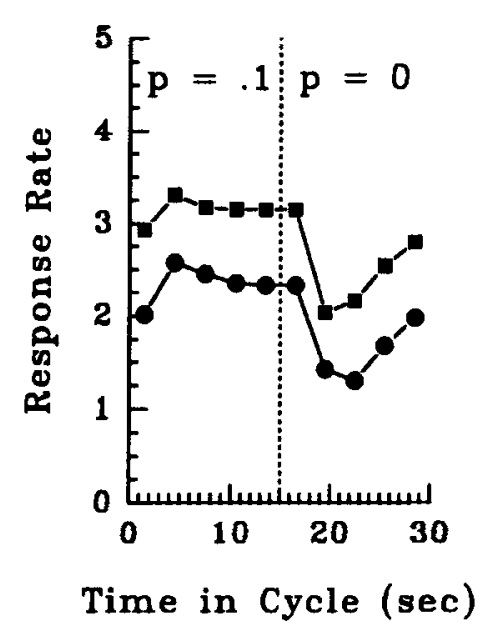

Figure 8. A simulation of the response rate for Pigeon 04 (closed squares) and Pigeon 08 (closed circles) in the $M=30$ condition using the response-rate profiles from Figure 6. Compare these simulations with the average response rates across the cycle for these animals in this condition in Figure 3. Note that response rates are not as great in the $p=.1$ part of the cycle as they are in Figure 3, but that other aspects of the simulation are a good match.

riod of extinction at $M / 2 \mathrm{sec}$ (half the cycle length) after a reinforcement, response rates, as seen in Figure 6, show a transient decrease. Reinforcements that occur close together in time will sum their effects on response strength during this decrease, potentially leading to lower rates of responding. Reinforcements that occur further apart will sum their effects after this decrease, leading to higher rates. Thus, responding following first reward in each $p=.1$ part of the cycle should be higher than following subsequent food deliveries.

Figure 10 shows the quantitative prediction of the $\mathrm{CI}$ model for first versus subsequent food deliveries in the $p=.1$ component for all four conditions for an average pigeon. For these predictions, we used the average $R$ function from our combined data and assumed that behavior at time $t$ was a function of just the previous two food deliveries, with each having an equal effect (i.e., $a_{0}=a_{1}=.5$; $a_{i}=0$ for $i>1$ ). Even in this simple form, the CI model predicts the general features of behavior seen in Figure 9. The response-rate functions for first versus subsequent food deliveries are similar in the $M=15$ condition, but diverge in the $M=30$ and $M=60$ conditions in much the same way as the data in Figure 9. (Data variability in the $M=120$ condition, because of the small number of instances of first food deliveries, makes comparison here problematic.) In no case does CI predict a substantial elevation of responding following subsequent food deliveries over that of first food deliveries. The reason the $\mathrm{CI}$ model predicts no effect in the $M=15$ condition is that by the time the animal has returned to responding after a subsequent reinforcement, the suppression in response strength profile created by the first reinforcement in the cycle has passed. Note that this prediction fits the data in Figure 9.
The CI model also predicts that animals should respond more following the second food delivery in the $p=.1$ component than following the third or any subsequent food delivery. As with first versus subsequent food deliveries, $\mathrm{CI}$ predicts that responding following a third reinforcement in a cycle should be suppressed more through more proximate food deliveries than are those for second food deliveries. Figure 11 shows these predictions for the $M=60$ condition, where the model makes the clearest predictions and there is a sufficient number of incidents of second and third food deliveries in the data to obtain reliable numbers. The model predicts that response rate following the second food delivery in a component will initially be higher than the rate following the third or any subsequent deliveries. After about $12 \mathrm{sec}$, however, the curves cross over and rate of responding following third+ food deliveries becomes higher than the rate following second food deliveries. Data from the $4 \mathrm{pi}-$ geons are shown in Figure 12. Although the details of the functions $(R$, in Equation 2) are different for each animal, 3 of the 4 show the crossover predicted by the CI model. Bird 11 shows little differentiation between the two curves, as would be predicted if $a_{2}$ were small or zero.

We conclude that the behavior of well-trained pigeons on probabilistic food schedules is inconsistent with the RTLO model but consistent with a temporal-control model in which each food delivery has a more or less fixed effect that cumulates across at least two successive food deliveries. Experiment 2 compares the RTLO model and the CI model in a different way.

\section{EXPERIMENT 2 Effect of a Postfood-Dependent Schedule}

Both the CI model and the RTLO model imply that the postfood profile of responding will be affected by the timing of previous reinforcements. According to the RTLO model, because of the accumulated effects of earlier reinforcers, response rate following a reinforcer embedded in a cluster of reinforcers should be higher than that following an isolated reinforcer. The reinforcement-density effect predicted by the RTLO model is obligatory, depending only on the memory parameter, $\alpha$. According to the CI model, the postfood profile of responding also cumulates across at least two successive reinforcers, but the effect predicted by the CI model depends on the form of the postfood profile of responding that develops under a given reinforcement distribution. For example, if a schedule generated a profile that had the form of a brief increase in response rate right after food followed by a long period of low-rate constant responding, then little cumulation might be observed, because the time between the latest food delivery and any previous food delivery would be longer than the brief period of increased responding following a reinforcement. Given this profile, aftereffects of reinforcers preceding the current one would be minimal-unless the time between the current and preceding reinforcer(s) fell within the short "high" period of the postfood profile. If all 


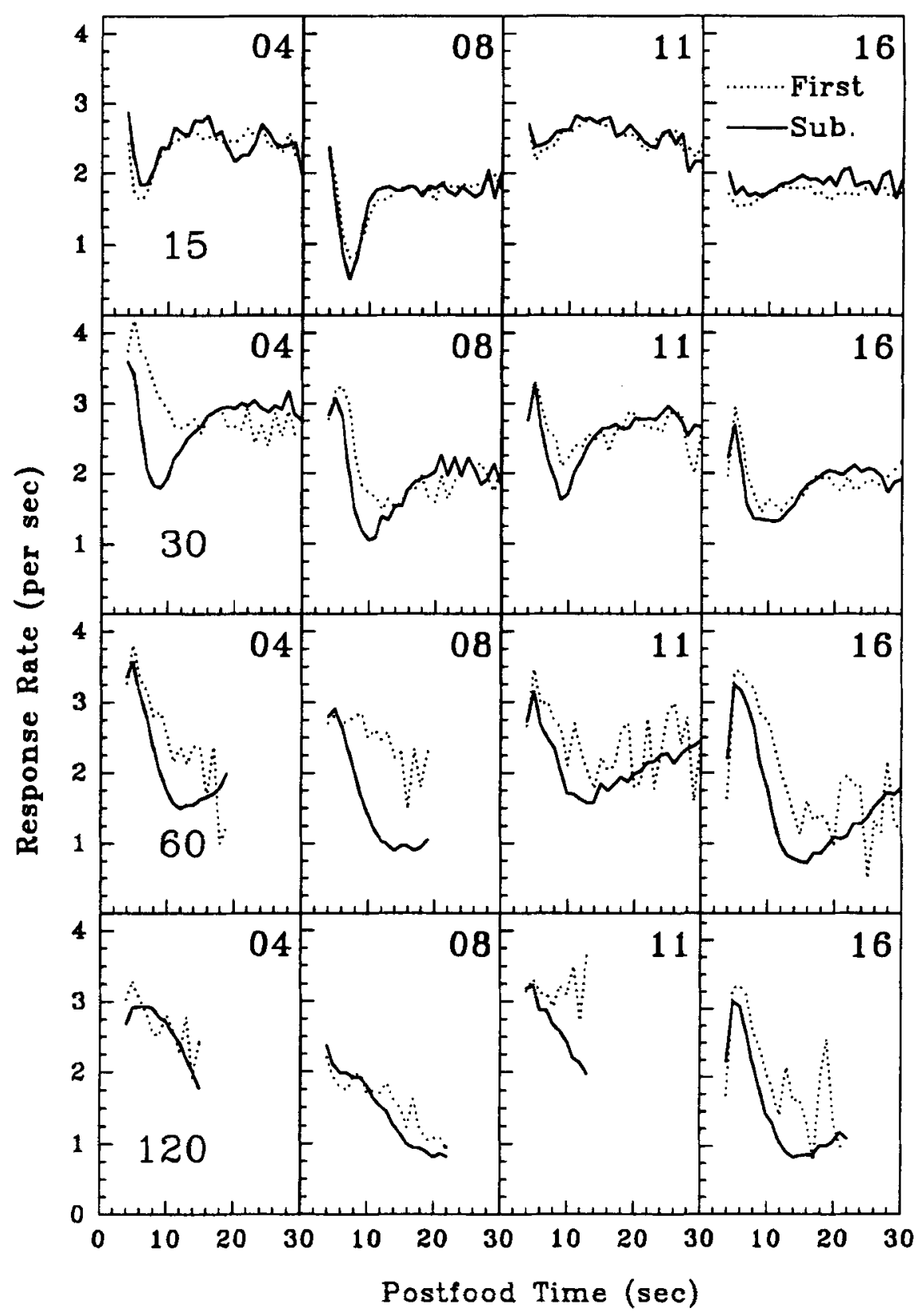

Figure 9. Average response rate in each second (first 4 sec excluded) of postfood time for all birds (columns) for the last five sessions of all conditions (rows). Dotted lines, average responserate profile following the first food delivery in each $p=.1$ part of the cycle; solid lines, average response-rate profile following all other food deliveries. As in Figure 6, to allow for food consumption, the first $4 \mathrm{sec}$ are not shown.

interfood intervals in a particular test schedule were longer than this brief period, no cumulation would be seen. Thus, for these kinds of schedules, the CI model would predict no effect of density and no differential effects from previous reinforcements. (This is the argument we made in the first experiment in condition $M=15$. In that condition, there was no difference between the responding following first and subsequent reinforcements-Figure 9-because the suppression in the response strength profile created by pre- vious reinforcements had already passed by the time the animal returned to responding after the latest reinforcement. See the prediction shown in Figure 10.) In Experiment 2 , we studied a schedule that generates a brief increase in response rate followed by a long period of low-rate constant responding in the postfood profile. Because of this peculiarity in the form of the postfood profile, the CI model and the RTLO model make very different predictions here. 

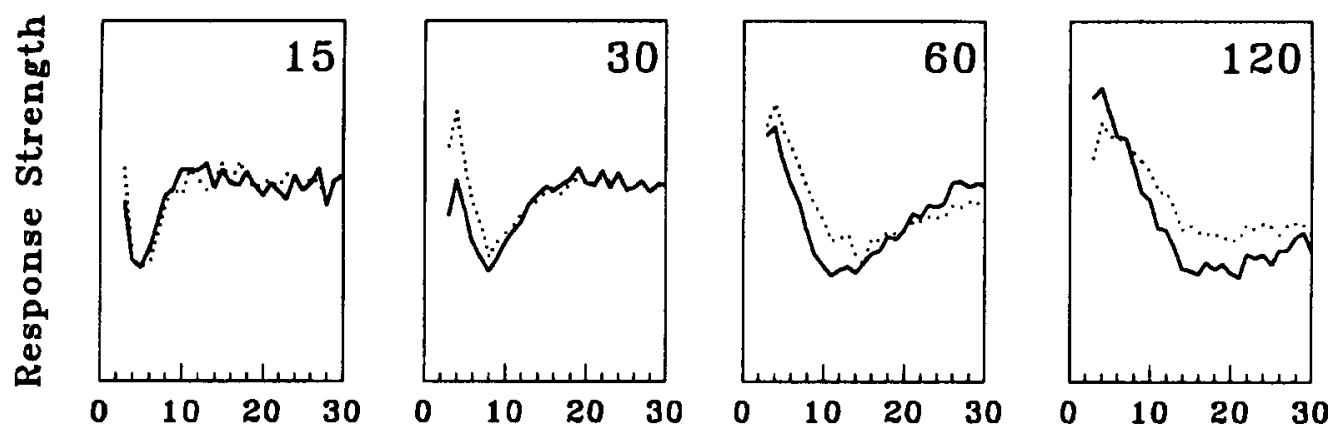

\section{Postfood Time (sec)}

Figure 10. Predictions of the cumulative impulse model for response strength as a function of postfood time following first versus subsequent food deliveries in the $p=.1$ part of the cycle for all four conditions. Dotted lines show the response-strength profile following the first food delivery; solid lines show the profile following subsequent food deliveries in the component. The response function for each prediction is based on the average response-rate profiles (in Figure 6) of all animals from the appropriate condition. Compare these predictions with the data in Figure 9.

\section{Method}

Subjects. Four White Carneaux pigeons (Columba livia), 3 males (Nos. 03, 07, and 08) and 1 female (No. 11), served as subjects. The animals were housed and maintained under the same conditions as in Experiment 1. All had had previous experience in learning experiments.

Apparatus. The apparatus was the same as in Experiment 1.

Procedure. As in Experiment 1, pecks to the center key were reinforced probabilistically (random ratio) with 2.5 -sec access to mixed grain. Similarly, all sessions ended when either the pigeon received 60 reinforcements or when $60 \mathrm{~min}$ had elapsed, whichever came first. The sessions were run once a day, usually for 6 days a week. Reinforcement probability in this experiment was dependent on postfood time. Pecks immediately following food were reinforced at a higher probability than those occurring at longer postfood times. The high and low probabilities were the same for all conditions, but the duration of the high period varied between conditions.

There were two types of sessions: (1) baseline sessions, in which pecks were reinforced according to a constant random-ratio 13.33

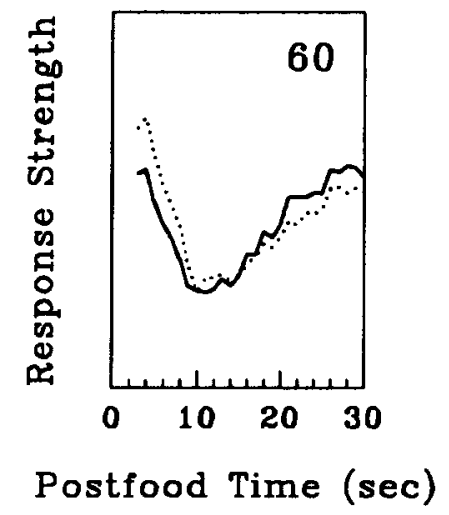

Figure 11. Response strength versus postfood time profile for the second (dotted line) versus third and any subsequent (solid line) food deliveries in the $p=.1$ part of the $M=60$-sec cycle, as predicted by the cumulative impulse model. The impulse function is based on an average of the response-rate profiles for all animals from Figure $6, M=60$ sec using $a_{0}=a_{1}=a_{2}=.333$. The first 4 sec are omitted. Other details are in the text. schedule ( $p=.075$ ), independent of postfood time; and (2) postfooddependent $(\mathrm{PD})$ sessions, in which pecks immediately following food were reinforced according to a random-ratio 8 (RR $8 ; p=.125)$ schedule. After a criterion postfood time $T$, however, pecks were reinforced at a lower probability $(\operatorname{RR} 40 ; p=.025)$. Each reinforcement reset the schedule's postfood clock, and $T$ was measured from the onset of food delivery.

The criterion time, $T$, varied over PD conditions: we studied $T=$ $4.36,5.02,5.90$, and $7.08 \mathrm{sec}$. All of these $T$ values are either less than or essentially equal to the effective interreinforcement interval and were chosen to produce a wide range of reinforcement densities. To minimize carryover effects, PD conditions were separated by baseline conditions and the sequence of PD conditions was counterbalanced. The sequence of conditions for the critical time, $T$ (in seconds), and the number of sessions in each condition for each bird are shown in Table 2. All PD conditions had baseline conditions both preceding and following. The number of PD and baseline sessions for all conditions is given in the bottom two rows of Table 2.

\section{Results and Discussion}

Figure 13 shows response rates for all 4 pigeons in each PD condition as a function of postfood time. With a few exceptions, the form of the response-rate function changes systematically as a function of the length of the high time, $T$. Performance in the baseline condition shows a small initial blip in response rates following a food delivery for 2 birds (No. 03 and especially No. 08 ). As predicted from temporal control and the CI model, rate of responding is high right after reinforcement and then drops off to a constant level for all animals in the 4.36-, 5.02-, and 5.90-sec conditions. The only exception is Bird 07 in the 4.36-sec condition. The postfood profile is flatter in the $7.08-\mathrm{sec}$ condition than in the shorter- $T$ conditions, and the high-low transition has little effect on the postfood response profile of Birds 07 and 11. This failure to detect the transitions in reinforcement probability for longer values of $T$ may reflect the limit of the animal's temporal discrimination ability in these schedules. Averaging reinforcement probability profiles over geometrically increasing bin sizes (as we 


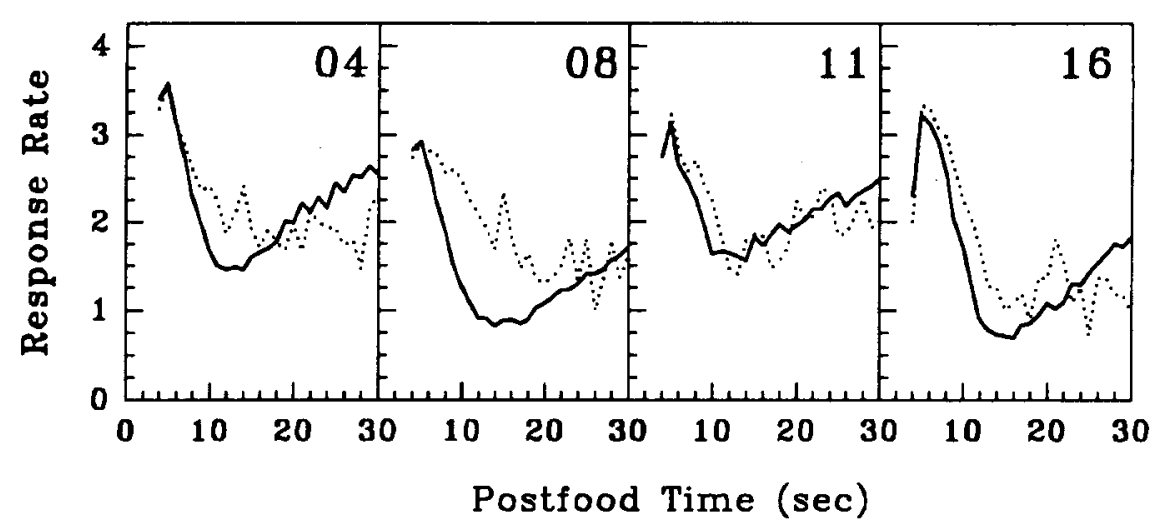

Figure 12. Response rate versus postfood-time profile for the second (dotted line) versus third and any subsequent (solid line) food deliveries in the $p=.1$ part of the $M=60$-sec cycle for the 4 pigeons. Compare with the prediction in Figure 11.

did in Figure 7) would tend to smooth this transition out for longer $T$ values.

The RTLO model predicts an effect of previous reinforcements on response rate; the CI model predicts an effect only if the $T$ value (duration of high reinforcement probability) is longer than the total effective interfood interval such that the elevated period of the postfood response profiles for the previous two reinforcers overlap. Because, on average, it takes $3.5 \mathrm{sec}$ from the onset of food delivery for a pigeon to return to responding, the minimum amount of time needed for a pigeon to pick up two reinforcers in succession is $7 \mathrm{sec}$. This total interfood interval is less than or essentially equal to the $T$ values used in this experiment, so CI would predict that there should be no effect of previous reinforcement and thus no effect of reinforcement density.

Do previous reinforcements have an effect in this experiment? In Experiment 1 (Figure 9), we found an elevation in response rate following the first reinforcement in a cycle (context of low reinforcement rate) compared with subsequent reinforcements (context of high reinforcement rate), consistent with the CI model and inconsistent with the RTLO model. Figure 14 shows a similar analysis for Experiment 2. The dotted lines show the postfood profile following reinforcements separated by more than $T \sec$ ( $T$ is the schedule parameter, $4.36 \mathrm{sec}$, $5.02 \mathrm{sec}$, etc.) from the preceding reinforcement (isolated reinforcements). The solid lines show the postfood profile of responding following the last reinforcer in a

Table 2

Sequence of Conditions for Each Bird in Experiment 2

\begin{tabular}{cccccccccc}
\hline & \multicolumn{7}{c}{ Sequence of Conditions } \\
\cline { 2 - 9 } Pigeon & BL & 1 & BL & 2 & BL & 3 & BL & 4 & BL \\
\hline 03 & & 4.36 & & 5.90 & & 7.08 & & 5.02 & \\
07 & & 7.08 & & 4.36 & & 5.02 & & 5.90 & \\
08 & & 5.90 & & 5.02 & & 4.36 & & 7.08 & \\
11 & & 5.02 & & 7.08 & & 5.90 & & 4.36 & \\
Cyclical & & 14 & & 14 & & 14 & & 14 & \\
Baseline & 3 & & 3 & & 3 & & 3 & & 2 \\
\hline
\end{tabular}

Note-BL, baseline. bout of reinforcers, where a bout is defined as a sequence of reinforcers with each separated by less than $T$ sec. These definitions are not critical, because Figure 14 shows that the postfood response profile is essentially the same after every reinforcement. This result is inconsistent with the RTLO model, but is predicted by CI, as outlined above.

Another implication of CI's prediction of no effect of previous reinforcements in this experiment is that reinforcement density should have no effect on response rates, whereas the RTLO model assumes that reinforcement density will have an effect on subsequent rates of responding. In this experiment, we used four $T$ values that we had calculated would give a wide range of bout lengths over the course of the experiment. Bout length (or the number of reinforcers in a bout) can be construed as a simple measure of reinforcer density in this experiment. Figure 15 shows response rates (averaged across all PD conditions and weighted per opportunity) as a function of bout length. As is evident from this figure, bout length has no effect upon response rate.

\section{GENERAL DISCUSSION}

Both experiments show that the RTLO model does not provide an accurate account of steady-state behavior on probabilistic reinforcement schedules. Gain and phase did not change in the predicted way with period of the input cycle in Experiment 1, and response rate was not elevated following bouts of reinforcement in either experiment. Temporal control, the idea that response rate tracks predictable postfood reinforcement probability, provided a better fit to the data in Experiment 1 but failed to account for an effect of previous reinforcement: an elevated postfood response profile following first reinforcements compared with subsequent reinforcements within a cycle. However, a modified version of the temporal control idea, the cumulative-impulse model, which allows cumulation of aftereffects across successive reinforcements, produced a closer approximation to experimental results in Experiment 1. 


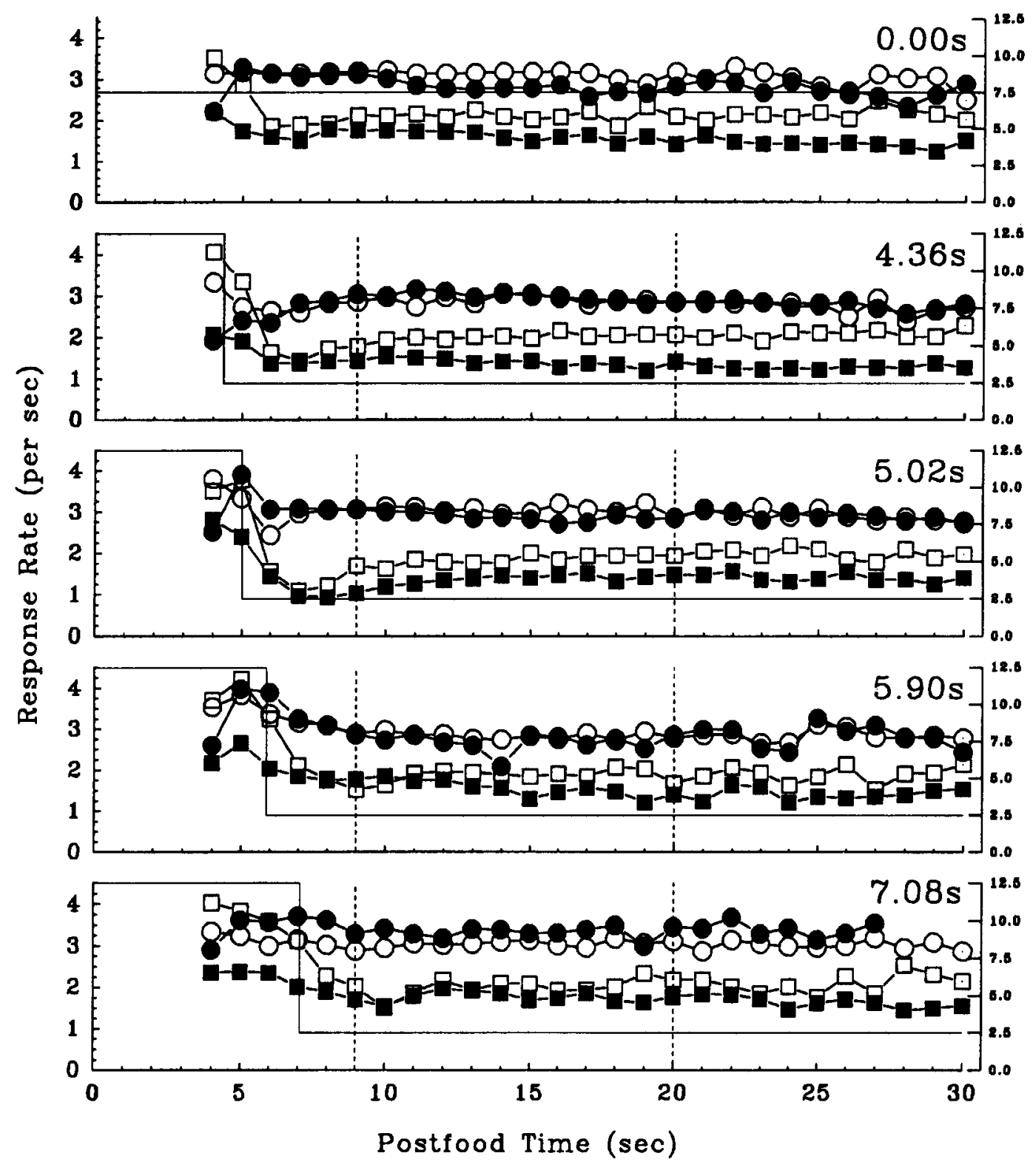

Figure 13. Response rates as a function of postfood time for all animals in all conditions in Experiment 2. Each data point represents response rate per second, per opportunity to respond during that postfood time. The first $4 \mathrm{sec}$ are omitted since postfood time is measured from the onset of food delivery. As new food delivery occurs, postfood time is reset. Solid lines show the reinforcement probability function as measured from the vertical axis on the right (in percent) and as a function of postfood time. (Vertical dashed lines indicate the area between which response rates are calculated for Figure 15.) Closed squares, Pigeon 03; closed circles, Pigeon 07; open squares, Pigeon 08; open circles, Pigeon 11.

The version of the CI model proposed in Experiment 1 accurately predicted the noneffect of previous reinforcements in Experiment 2. For Experiment 2, the CI model makes the counterintuitive prediction that previous reinforcements will have no influence upon responding as long as the $T$ value (the duration of a high-probability postfood period) is less than it takes to acquire and consume two successive reinforcements (approximately $7 \mathrm{sec}$ ). The data in this experiment show essentially iden- tical postfood profiles of responding following isolated versus the last reinforcers in a bout, thus confirming CI's prediction. This finding and the similar finding that bout length has no effect upon response rates in this experiment is counter to what would be expected from the RTLO model.

Experiment 2 could have been a stronger test of the CI model had we also included $T$ values greater than $7 \mathrm{sec}$, thereby allowing the facilitatory effects of previous re- 


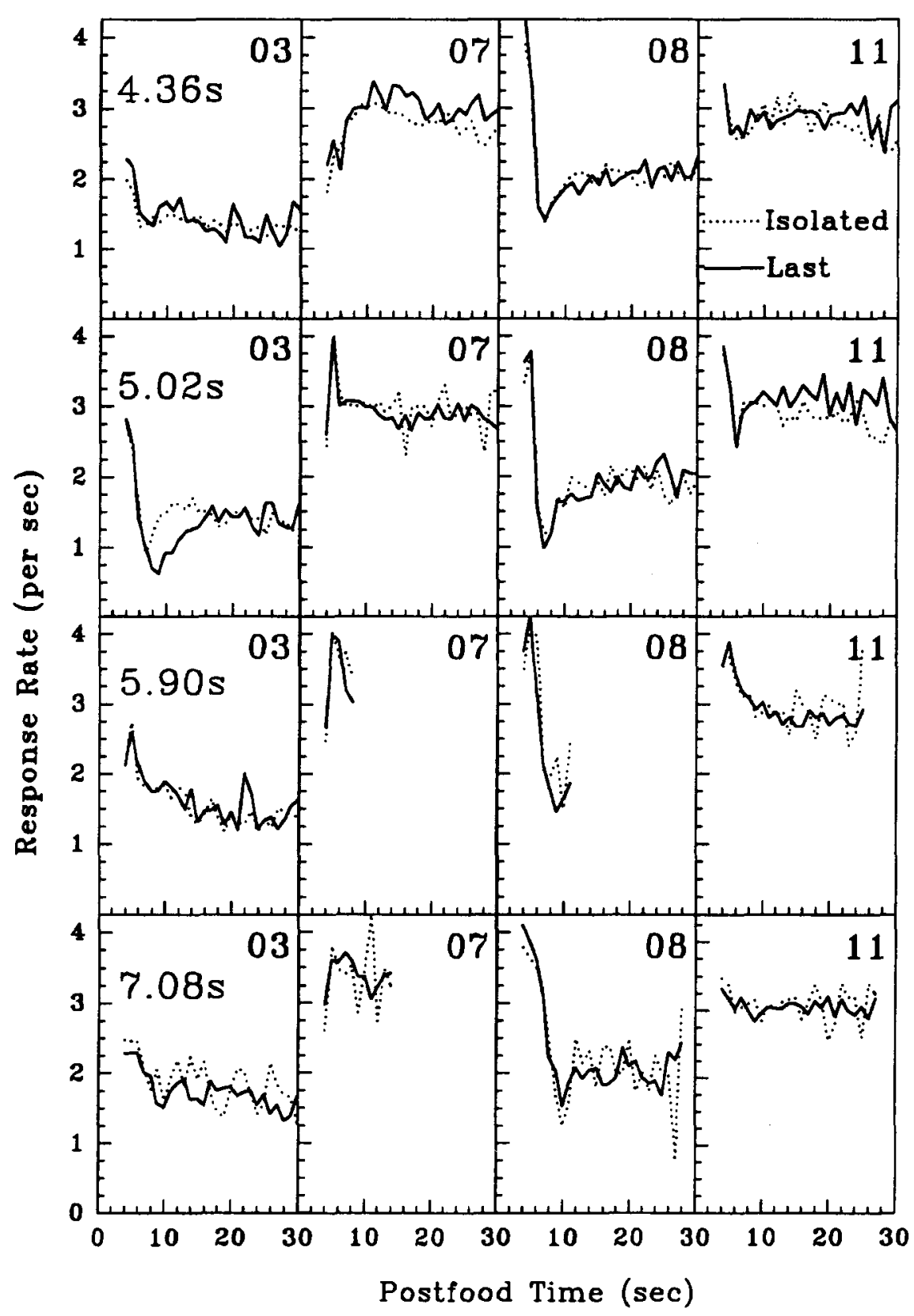

Figure 14. Average response rate in each second of postfood time for all birds (columns) for the last five sessions of all conditions (rows). Dotted lines show average response-rate profile following the only food delivery in a bout (isolated reinforcement); solid lines show average response-rate profile following the last reinforcement in a bout of multiple food deliveries. The first $\mathbf{4}$ sec are omitted in this figure.

inforcements to cumulate over time and hence develop "integration-like" performance. This logical test of the model was not undertaken for two reasons. First, longduration $T$ values would mean that more and more reinforcements would have occurred in bouts. At $T=7 \mathrm{sec}$, almost all bouts were of more than 4 and 5 reinforcements in length, and our calculations lead us to believe that $T$ values greater than $8 \mathrm{sec}$ would have resulted in gen- erally one long bout per session. Second, the tendency of the animals not to discriminate the transitions in reinforcement probability at higher $T$ values (see Figure 13), indicates that longer $T$ values may not be discriminable at all, making the comparison moot for the CI model.

Given that the RTLO model fits our data so poorly, it is worth asking why it has proved so popular in foraging theory. One reason is surely analytic convenience. But 


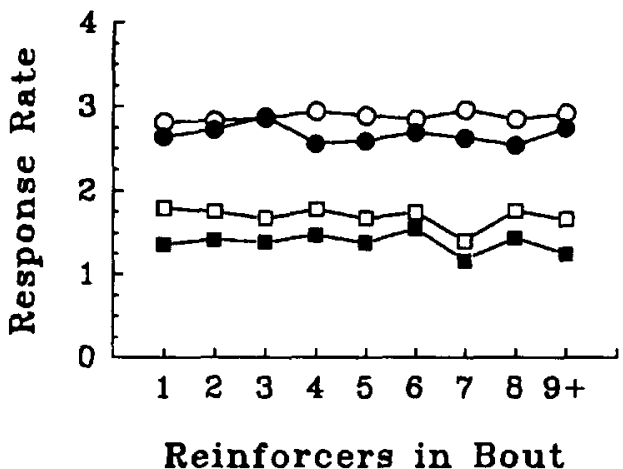

Figure 15. Response rate as a function of bout length. Response rate is taken as an average rate of responding over the interval 9-20 sec postfood delivery (see Figure 13) to provide for a sufficiently short period of time after a reinforcer in which we might expect change in the animal's estimation of reinforcement probability to have an influence (i.e., $<20 \mathrm{sec}$ ), while subtracting out any transient influences from a single reinforcement (i.e., $<9$ sec).

there may be another. In the steady state, when the animal has had sufficient opportunities to learn about the pattern of postfood reinforcement probability, behavior is consistent with temporal control, supplemented by the idea of partial cumulation across reinforcers. But early in training, the animal must presumably rely on a default postfood reinforcement probability, which data show to be very much like the RTLO model: each reinforcer produces a burst of operant behavior that slowly extinguishes until the next reinforcement delivery. (See, e.g., Ferster \& Skinner's, 1957, stylized cumulative record of fixedinterval acquisition and the data from our own baseline conditions.) This postfood bursting is just a temporal version of area-restricted search. Therefore, the popularity of the temporal-control interpretation in the operant literature may simply reflect a focus on steady-state data (and intermittent-reinforcement schedules). The popularity of the RTLO model in the foraging literature may reflect a more transient exposure of subjects to the experimental procedure and a focus on less temporally predictable reinforcement schedules.

\section{REFERENCES}

Bobisud, L. E., \& Voxman, W. L. (1979). Predator response to variation of prey density in a patchy environment: A model. American Naturalist, 114, 63-75.

Bush, R. R., \& MOSTELLER, F. (1951). A mathematical model for simple learning. Psychological Review, 68, 313-323.

CATANIA, A. C. (1970). Reinforcement schedules and psychophysical judgments: A study of some temporal properties of behavior. In W. N. Schoenfeld (Ed.), The theory of reinforcement schedules (pp. 43-61) New York: Appleton-Century-Crofts.

Catania, A. C., \& Reynolds, G. S. (1968). A quantitative analysis of the responding maintained by interval schedules of reinforcement. Journal of the Experimental Analvsis of Behavior, 11, 327-383.

Charnov, E. L. (1976). Optimal foraging: The marginal value theorem. Theoretical Population Biology, 9, 129-136.

Commons, M. L., Woodford, M., Boitano, B. A., Ducheny, J. R., \& PECK, J. R. (1982). Acquisition of preference during shifts between terminal links in concurrent chain schedules. In M. L. Commons, R.A. Herrnstein, \& A. R. Wagner (Eds.), Quantitative analyses of behavior: Vol. III. Acquisition (pp. 391-426). Cambridge, MA: Ballinger.

FERSTER, C. B., \& SKInNER, B. F. (1957). Schedule of reinforcement. Englewood Cliffs, NJ: Prentice-Hall.

GIBBON, J. (1977). Scalar expectancy theory and Weber's law in animal timing. Psychological Review, 84, 279-325.

HARLEY, C. B. (1981). Learning the evolutionarily stable strategy. Journal of Theoretical Biology, 89, 611-633.

HERRNSTEIN, R. J. (1961). Relative and absolute strength of response as a function of frequency of reinforcement. Journal of the Experimental Analysis of Behavior, 4, 267-272.

HeRrnsteIN, R. J. (1990). Rational choice theory: Necessary but not sufficient. American Psychologist, 45, 356-367.

Herrnstein, R. J., \& VAughan, W. (1980). Melioration and behavioral allocation. In J. E. R. Staddon (Ed.), Limits to action: The allocation of individual behavior (pp. 143-176). New York: Academic Press.

HoRNer, J. M. (1991). A single board computer for the control of operant experiments. Behavior Research Methods, Instruments, \& Computers, 23, 513-522.

KACELNIK, A., KREBS, J. R., \& ENS, B. (1987). Foraging in a changing environment: An experiment with starlings. In M. L. Commons, A. Kacelnik, \& S. Shettleworth (Eds.), Quantitative analyses of behavior: Vol. IV. Foraging (pp. 63-87). Hillsdale, NJ: Erlbaum.

KELLO, J. E. (1972). The reinforcement-omission effect on fixed-interval schedules: Frustration or inhibition? Learning \& Motivation, 3, 138147.

KILLEEN, P. R. (1982). Incentive theory. In D. Bernstein (Ed.), Nebraska symposium on motivation (pp. 169-216). Lincoln: University of Nebraska Press.

LEA, S. E., \& Dow, S. M. (1984). The integration of reinforcement over time. In J. Gibbon \& L. Allan (Eds.), Time and time perception (Annals of the New York Academy of Sciences, Vol. 423, pp. 269-277). New York: New York Academy of Sciences.

LESTER, N. (1984). The feed-feed decision: How goldfish solve the patch depletion problem. Behavior, 89, 175-199.

Mellon, R. C., Leak, T. M., Fairhurst, S., \& Gibbon, J. (1995). Timing processes in the reinforcement omission effect. Animal Learning \& Behavior, 23, 286-296.

Myerson, J., \& Miezin, F. M. (1980). The kinetics of choice: An operant systems analysis. Psychological Review, 87, 160-174.

Ollason, J. G. (1980). Learning to forage optimally? Theoretical Population Biology, 18, 44-56.

Pulliam, H. R., \& Dunford, D. (1980). Programmed to learn: An essay on the evolution of culture. New York: Columbia University Press.

RegelmanN, K. (1984). Cooperative resource sharing: A simulation model. Animal Behaviour, 32, 226-232.

SCHNEIDER, B. A. (1969). A two-state analysis of fixed-interval responding in the pigeon. Journal of the Experimental Analysis of Behavior, 12, 677-687.

STADDON, J. E. R. (1965). Some properties of spaced responding in pigeons. Journal of the Experimental Analysis of Behavior, 8, 19-27.

STADDON, J. E. R., \& INNIS, N. K. (1966). An effect analogous to frustration on interval reinforcement schedules. Psychonomic Science, 4, 287-288.

(Manuscript received February 27, 1995; revision accepted for publication March 19, 1996.) 